\title{
Radiation Sources in Structural Biology
}

\author{
Richard Henderson $^{a \star}$, Mejd Alsari $^{\mathrm{b}}$
}

\begin{abstract}
What is radiation damage? Are electrons more suitable than X-rays in structural biology? Richard Henderson talks about synchrotron radiation and how cryo-EM laboratories are being established at synchrotrons as national research facilities. See video at https://youtu.be/CQ-Gz9daVL8
\end{abstract}

Mejd Alsari (MA). You also wrote a review on radiation damage where you compare different radiation sources, electrons, neutrons, and x-rays. ${ }^{1}$ Can you tell us a bit about that paper and what was the motivation?

Richard Henderson (RH). In the group I was part of we were trying to push in different directions. We knew what the weak points were and we were working on them. In a way that review was a sort of slight diversion. In parallel with the development of all the electron cryo-microscopy methods, which always looked like it had a lot of potential, there were other people developing other methods.

For example X-ray crystallography had originally used electrons to produce the X-rays by firing electrons into a piece of copper and then getting the X-rays out, shine them at crystals, and get patterns. That was back in the 30s. Then they wanted brighter X-ray sources so they developed rotating anodes, where you made the anode move so that it didn't melt when you put the beam in and you get more $\mathrm{X}$-rays. But eventually it was discovered that you could get much brighter, millions of times brighter, X-rays out of the synchrotron radiation that comes from electrons going around in circles. Just because they're being accelerated they produce X-rays and they're very powerful. So probably about 1980, there was the first synchrotron dedicated to producing X-rays rather than doing particle collisions, in Daresbury in the UK. ${ }^{2}$

Then Europe got together and decided to build one in Grenoble that's the European Synchrotron Radiation Facility (ESRF). ${ }^{3}$ Once they built the synchrotron they'd have to decide what kind of cameras and what kind of specimens were going to look at. One of the things synchrotrons can do very well is X-ray microscopy.

There was a meeting at Grenoble that was run by Andrew Miller and Carl-Ivar Brändén, who were the biological sciences director and ex-director, they were running the meeting and they wanted to know whether it's a good idea to build an X-ray microscope. Because I had come from X-ray crystallography and I was doing electron microscopy we knew all about radiation damages and so on. I had published papers about it before. So I was asked to go along and give a talk explaining about how much radiation damage you get with electrons how much you get with X-rays. The bottom line was that electrons are 1,200 times better than X-rays. I gave this talk and there were 50 people in the audience, they all were $\mathrm{X}$-ray microscopy people, they were really very angry because they wanted 5 or 10 million euros to build their synchrotron beamline for X-ray microscopy and I'm telling it's a really bad idea. You can do quite good X-ray microscopy, not on molecules and atoms, but on cells and so on. ${ }^{4}$

They did get their beamline in the end. Because they didn't understand this I thought "Well, you know, if fifty clever physically oriented scientists don't know this, perhaps it would be a good idea to write a review". I thought I would write a review just comparing
$\mathrm{X}$-rays and electrons in terms of radiation damage and resolution, although I published papers before about it, obviously they hadn't noticed them.

So I wrote this review and then during that time there's another journal called Neutron News. We used to do some neutron diffraction as well. That's slightly useful in structural biology. Because we did one experiment and published one paper in 1984, every few months I still get a journal called Neutron News. While I was writing this review about X-rays and electrons, Neutron News arrived with big full-scale table with every single element, every single isotope, telling you its lifetime, and how it interacted, neutron cross-sections, elastic, inelastic and so on. ${ }^{5}$ I thought maybe I should expand it to be electrons, X-rays, and neutrons. My surprise was that not only are electrons 1,200 times less damaging for the information you get in the images than X-rays, they are also three times less damaging the neutrons, even though neutrons have a very weak interaction. So I thought that was very good.

After having written that and published it I thought well maybe we should, since electrons are so clearly better, do some more calculations. ${ }^{1}$

If you then now focus on electrons, what is the genuine theoretical potential? Assuming all practical problems, which is what we've been working on, microscopes, vacuum, sources, detectors, supposing they all work perfectly and we had phase plates and so on, how well would it do? It came out that you do very well. So after writing the review for a different purpose, we decided we would give up all crystallography and switch entirely into the single particle electron cryo-microscopy. So from about 1995-96 that's what we did.

For example people came to work (with us), there was a visitor called Sriram Subramaniam, who now does electron cryo-microscopy here came and we said "Okay in one year we're going to do 10 structures, all at atomic resolution, by electron cryo-microscopy" and then we started. But we discovered of course that quite a lot of problems that needed to be solved and that took another 15 years or something like that.

Essentially the vision was there and the theoretical underpinning was there in that review. So even though it was done for a different purpose it actually had served a number of purposes afterwards.

MA. Do you think we still need synchrotrons for structural biology, perhaps for small molecules?

RH. Yes and not only for small molecules actually. They have been greatly improved. The intensity of the X-ray beams or any electromagnetic radiation you get out of synchrotrons compared to 1980 , has been increased many million fold. The quality of the $\mathrm{X}$-rays you get out of synchrotrons is really fantastic now and it's still being improved. Nearly all the synchrotrons in the world, the state-of-the-art ones, have been running for nearly 20 years, periodically they shut them down and they rebuild the magnets and the focusing elements to make them brighter and brighter. At the

a MRC Laboratory of Molecular Biology, Cambridge UK, ${ }^{\text {a }}$ Cavendish Laboratory, University of Cambridge, UK (ma671@cam.ac.uk) 
moment the ESRF, the same one that I went to that meeting, is currently being upgraded, which will give them probably a factor of 100 improvement in the intensity. ${ }^{6}$ That means that if you have a crystal, let's say, in 1960s it would take you months to collect one three-dimensional data set of all the diffraction from the different angles in a crystal. You can do that now in one second instead of months. If you have a crystal of a biological structure you're interested in, you can solve it in minutes now.

One of the big uses of X-ray crystallography at the moment is in the development of new small molecule drug, let's say, an oral pill that you take to cure you of stomach ulcers or heart problems and so on. Usually the biological target would either be a human protein or a bacterial protein (in the case of antibiotics). If you're analysing that protein, you make a crystal of the protein, you can then add to the crystal the drug that you're trying to develop and determine in a minute or so the structure of the enzyme with your small molecule binding to it. That small molecule might either inhibit, block, the activity of that biological molecule or it could activate it depending on what it is that you're trying to do.

Then once you've got one molecule, the pharmaceutical companies usually make hundreds if not thousands before they get one that has all of the qualities that they're looking for to make a really good treatment for some human disease or ailment and so on. So the structural biologists would like to determine the structure of hundreds of these compounds.

The great thing about synchrotrons is that the brightness of the $\mathrm{X}$-ray beams is so big. The detectors are quite good, they can always be improved. They can do 300 structures every day, whereas with electron cryo-microscopy at the moment it's about one structure per day.

Now, obviously, you can make detectors bigger and faster and the electron microscopy will get better and better, but you'll still have this advantage that once you've got a crystal it's very quick to do different complexes with different small molecule ligands.

Synchrotrons will just get brighter and brighter. Probably we have enough of them. It's just that they all need to be continually improved. One of the new directions that have been developed is instead of having a synchrotron that makes all the electrons go in a circle and produces X-rays coming out in all directions or other electrical radiation coming out in all directions, there are now linear free electron laser synchrotrons, ${ }^{7}$ where you accelerate the beam using a radio frequency magnetic wave to accelerate electrons and as they as they go faster and faster with this electromagnetic wave you get, along with the electrons, $\mathrm{X}$-rays that go with them and then coming out of the end you have a brighter and more powerful X-ray beam and an electron beam (compared to standard synchrotrons). Of course the electrons you can just divert them away. You get a pure pulsed electron beam and these are even brighter than what they call third-generation synchrotrons. It's a technique and a brighter source looking for problems, where it can be used to solve them.

So synchrotrons will never go away they'll always be needed and in structural biology electron cryo-microscopy is still not producing every year, let's say, for every electron cryo-microscope structure that is deposited in the public databases, of course it couldn't be done by any other method, you're getting at the moment eight or nine structures being deposited by X-ray crystallography although many of them are sort of related structures. They are often repeats with slightly different ligands.

So the idea is that as the methods improve the X-rays will get brighter, the electrons will get faster, the detectors will get better. We think if you look at the statistics and how people are deciding to spend their time in the structure biology field it looks like in about four or five years the number of structures being deposited will be about the same from electron cryo-microscopy and X-ray crystallography (Figure 1).

Then after that, that's five years from now, we already know prob-

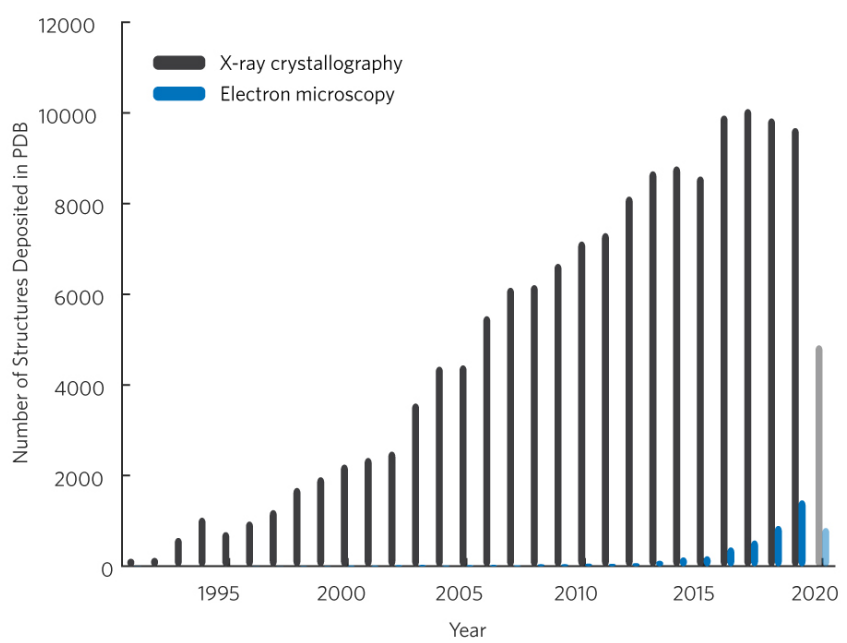

Figure 1 Number of entries deposited in the Protein Data Bank per Year (05 June 2020).

ably about half of the structures in biology, we have atomic models either for the structure you're interested in or a very closely related one. So probably within five years you'll find that most of the landscape of structural biology has been mapped. There are in the databases examples of nearly all the biological structures you'll be interested in. For example bacteriorhodopsin now. There was one structure in $1990 .{ }^{8}$ Now in if you look in the database, there's 200 of them with different ligands, different mutants, different intermediates trapped and many of the enzymes, you'll find that there's a thousand different examples of them. ${ }^{9}$

As the methods get more powerful, instead getting one structure in years, now you can look at the structure, how it behaves, what its mechanism is, how it interacts with other molecules, and so on. There will be a broadening of the landscape and the ambitions of the structural biologists to tackle problems in addition to just finding out what the basic structure is.

MA. What do you think about facilities such as the CM01 (ESRF), ${ }^{10}$ eBIC (Electron Bio-Imaging Centre, Diamond Light Source) ${ }^{11}$ and ePSIC (electron Physical Science Imaging Centre, Diamond Light Source $)^{12}$ how do they play a role in the community?

RH. As the use of X-ray diffraction grew in the structural biology community both for structural biology and for chemistry, physics, materials science and so on, it became clear that people couldn't have a synchrotron in every lab because they cost $£ 400$ million to build. ${ }^{13}$ From about the 1980 s or 1990 s the idea of having national facilities came about. In the UK we have one synchrotron, France has one synchrotron, Germany has one synchrotron, Europe has a bigger synchrotron, America has about three or four, Japan has two or three, and so on. There are probably 20 or 25 . Italy has one and so on. There are synchrotrons in the world that are all national or regional facilities.

In the UK, they were probably the first ones, two scientists here Dave Stuart, based in Oxford, and Helen Saibil, based in London, about 10 years ago they decided actually that electron microscopy was also beginning to become more expensive. They couldn't justify one microscope costing $\mathfrak{E} 5$ million in every lab. So they advocated and succeeded in getting funding, some grants, from the Wellcome Trust, which is a UK-based charity, to buy the first high-end 300 $\mathrm{keV}$ electron cryo-microscope. It was going to be based at the synchrotron (Diamond Light Source). They would use the management system they had developed over 10 years for applying for access to the synchrotron beam lines. They'd used the same thing for electron microscopy. Particularly Dave Stewart advocated, as part of an upgrade to the synchrotron, where they were building one long beamline (one of the 30 beam lines was a long beamline), at the end 
station for a long coherent X-ray beam line, the construction of a big building that was also for electron microscopy. It's several thousand square meters and half of it was supposed to be for electron microscopy tied to physical sciences. That's ePSIC. The other half was tied to the biological sciences which Dave Stewart and Helen Saibil had been. When the Wellcome Trust funded the first microscope said you cannot have a national facility with one microscope because if it goes wrong it won't work, you need two. So they were persuaded that they applied for one and they were given two. ${ }^{14}$

Now of course, because it's such a successful and popular method, two is not enough and so they are now up to five plus one or two other microscopes so they've got about seven or eight in eBIC. It is now quite a successful fully integrated with the synchrotron, the same beam line allocation panels.

So the idea now I think is it that there are very expensive microscopes that are successful and many people, who are just the users, don't want to know anything about them. They just want "here is my protein, I want the structure" and they don't want to know anything else.

These national facilities they're here to stay and many countries have decided to co-locate them within the synchrotrons because of the common underlying infrastructure to manage it. Stanford in the USA has got an EM centre now alongside its synchrotron. ${ }^{15}$ Brookhaven in the East Coast of America are thinking of doing that.

But there are also national centres for EM that are not at synchrotrons and, again, we don't know quite how things will develop but it's a good model of basing them at synchrotrons. Here in this lab we have three of these high-end microscopes and we are debating is it a good idea for us to be running a big facility like that or should we integrate it with other national facilities. Because the UK has been a leader in this type of electron microscopy, or one of the leaders, there are now 20 or 21 of these high-end microscopes in the UK (two in Leeds, one in Glasgow, one in Leicester, and so on). They are distributed, but the biggest number are in Oxford, where they have six or seven of them there.

\section{References}

1 R. Henderson. The potential and limitations of neutrons, electrons and X-rays for atomic resolution microscopy of unstained biological molecules. Quarterly Reviews of Biophysics 28, 171-193, (1995).

2 Wikipedia. Synchrotron Radiation Source, https://en.wikipedia.org/wiki/Synchrotron Radiation Source, (2020).

3 ESRF. ESRF, https://www.esrf.eu/, (2020).

4 R. Henderson. From Electron Crystallography to Single Particle CryoEM (Nobel Lecture). Angewandte Chemie International Edition 57, 10804-10825, (2018).

5 V. F. Sears. Neutron scattering lengths and cross sections. Neutron News 3 , 26-37, (1992).

6 ESRF. ESRF-EBS, https://www.esrf.eu/about/upgrade, (2020).

7 E. Jaeschke, S. Khan, J. R. Schneider \& J. B. Hastings. Synchrotron Light Sources and Free-Electron Lasers, https://link.springer.com/referencework/10.1007\%2F978-3-319-04507-8\#toc, (2019).

8 R. Henderson, J. M. Baldwin, T. A. Ceska, F. Zemlin, E. Beckmann \& K. H Downing. Model for the structure of bacteriorhodopsin based on high-resolution electron cryo-microscopy. Journal of Molecular Biology 213, 899-929, (1990).

9 P. D. Bank. Search: bacteriorhodopsin, https://www.rcsb.org/, (2020).

10 ESRF. CMO1 CRYO-ELECTRON MICROSCOPE, https://www.esrf.eu/ home/UsersAndScience/Experiments/MX/About our beamlines/CM01. html, (2020).

11 D. L. Source. eBIC: Electron Bio-Imaging Centre, https://www.diamond ac.uk/Instruments/Biological-Cryo-Imaging/eBIC.html, (2020).

12 D. L. Source. electron Physical Science Imaging Centre (ePSIC), https://www. diamond.ac.uk/Instruments/Imaging-and-Microscopy/ePSIC.html, (2020).

13 D. L. Source. General Questions, https://www.diamond.ac.uk/Home/About/ FAQs/General.html, (2020).
14 D. L. Source. Funding announced for new biological facilities at Diamond, https://www.diamond.ac.uk/Home/News/LatestNews/16-12-13.html, (2013).

15 SLAC. Stanford-SLAC Cryo-EM Facilities, https://sites.slac.stanford.edu/ cryo-em/, (2020).

\section{Conflicts of Interest}

There are no conflicts to declare.

\section{Acknowledgements}

M.A. thanks Khalaf Al Habtoor, chairman of Al Habtoor Group, for sponsoring the video production. M.A. also thanks the MRC Laboratory of Molecular Biology for arranging the interview with R.H.

\section{Author Contributions}

R.H. was interviewed by M.A., who wrote this manuscript.

\section{Keywords}

Structural biology, cryo-EM, Richard Henderson, synchrotron, radiation damage.

\section{Article Information}

This article is licensed by R.H. et al. under a Creative Commons Attribution 4.0 International License (CC BY 4.0). 